\title{
Interacciones tróficas de las seis especies de peces más abundantes en la pesquería artesanal en dos bahías del Pacífico Central Mexicano
}

\author{
J.R. Flores Ortega ${ }^{1 *}$, E. Godínez Domínguez ${ }^{1}$, J.A. Rojo Vázquez ${ }^{1}$, A. Corgos ${ }^{1}$, V.H. Galván \\ Piña $^{1} \&$ G. González Sansón ${ }^{2}$ \\ 1. Departamento de Estudios para el Desarrollo Sustentable de Zonas Costeras, Universidad de Guadalajara. V. Gómez \\ Farias \# 82, San Patricio-Melaque, Jalisco, México. 48980. Tel: (315) 355-6330, Fax: (315) 355-6331; \\ percebes5@gmail.com \\ 2. Centro de Investigaciones Marinas, Universidad de La Habana, La Habana, Cuba. \\ * Autor de correspondencia
}

Recibido 08-VI-2009. Corregido 08-VIII-2009. Aceptado 08-IX-2009.

\begin{abstract}
Trophic interactions of the six most abundant fish species in the artisanal fishery in two bays, central Mexican Pacific. We surveyed the trophic components in six species of Bahía de Navidad and Bahía de Chamela: Microlepidotus brevipinnis, Caranx caballus, Haemulon flaviguttatum, Lutjanus guttatus, L. argentiventris and Mulloidichthys dentatus. Two main seasonal periods were considered: 1) North Equatorial Counter Current NECC period influence (T1) and 2) California Current CC period influence (T2). In Bahía de Navidad 78 prey taxa were identified in the stomachs. From July to December (T1), 64 prey taxa were found, and from January to June (T2), 45 prey items. In Bahía de Chamela 93 prey items were identified; 74 during T1 and 60 during T2. The highest prey number was found in the stomachs of M. dentatus during T1 in Bahía de Navidad and the lowest prey number (7) was recorded in H. flaviguttatum in Bahía de Navidad in the same period. Crustaceans were the most frequently recorded prey items, followed by fishes, mollusks, polychaetes, and echinoderms in both seasonal periods and sites. The six fish species studied are considered as specialist feeders due the low values of the niche breadth index. There was little similarity among the diets. Rev. Biol. Trop. 58 (1): 383-397. Epub 2010 March 01.
\end{abstract}

Key words: feeding ecology, tropical fish, hydroclimatic patterns, niche breadth, partitioning habitat.

La alimentación responde a un complejo sistema de adaptaciones y estructuras que facilitan la explotación de los recursos disponibles en el medio, y el objetivo es obtener el máximo de energía neta necesaria para el crecimiento y la reproducción, entre otras actividades propias de cada especie (Granado-Lorencio 2002). En función del tamaño y de las capacidades de los depredadores para capturar su alimento, la variedad de las presas encontradas puede ser muy amplia, y estar determinada por la disponibilidad de las presas, las cuales están asociadas a diferentes respuestas bióticas y abióticas en el ecosistema (Pratchett et al. 2004, Berumen et al. 2005). Los cambios en el ecosistema provocan el desarrollo de nuevas adaptaciones y estrategias en los peces para aprovechar los recursos disponibles y no limitarse a consumir sólo los preferidos (Pratchett \& Berumen 2008).

La descripción de las redes tróficas marinas provee información valiosa para conocer la estructura de la comunidad y del ecosistema (Link et al. 2006). En la actualidad las relaciones tróficas son comúnmente estudiadas por medio del análisis del contenido estomacal, una herramienta utilizada tradicionalmente para describir los componentes, niveles tróficos, y las relaciones depredador-presa (Gerking 1994).

Las complejas historias de vida de los depredadores pueden influir en todo el ecosistema 
ya que a menudo presentan migraciones entre hábitats por cambios ambientales y estacionales (Blaber 1997), cambios fisiológicos y morfológicos en los juveniles (Helfman et al. 1982, Gerking 1994, Hyndes et al. 1997), cambios en la distribución espacial del alimento y estrategias de alimentación (Edgar \& Shaw 1995a, b, Muñoz \& Ojeda 1998). Una estrategia implementada por los organismos para optimizar los recursos de un hábitat es la "partición de recursos", definida por Ross (1986) como cualquier diferencia sustancial en el uso de recursos entre especies que coexisten.

El presente trabajo analiza la amplitud del nicho y la superposición de las dietas de las seis especies de peces más abundantes capturadas por la pesquería artesanal de redes de enmalle en dos bahías próximas, pero con importantes diferencias oceanográficas y de hábitats, durante los dos principales periodos hidroclimáticos en el área.

\section{MATERIALES Y MÉTODOS}

Área de Estudio: Esta región tiene importancia por ser un sitio de convergencia de dos sistemas de corrientes importantes como son la Corriente de California (CC) y la Contracorriente Norecuatorial (CCNE) que producen cambios en los patrones de circulación debido a la dominancia de una de las dos corrientes en el área (Pacheco 1991, Filonov et al. 2000). Dentro de esta región están situadas la Bahía de Navidad (19¹0'30"

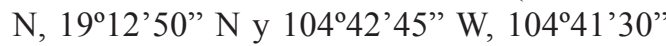
W) y la Bahía de Chamela $\left(19^{\circ} 21^{\prime} 11^{\prime \prime} \mathrm{N}\right.$, $19^{\circ} 38^{\prime} 06^{\prime}$ " N y $\left.105^{\circ} 01^{\prime} 43^{\prime \prime} \mathrm{W}, 105^{\circ} 12^{\prime} 30^{\prime \prime} \mathrm{W}\right)$, cada una con características físicas y ambientales particulares.

La Bahía de Navidad presenta una línea de costa irregular de aproximadamente 12 $\mathrm{km}^{z}$ de extensión con accidentes fisiográficos importantes: la laguna de Barra de Navidad en el extremo sur, y dos playas arenosas, delimitadas por acantilados. El lecho marino presenta fondos rocosos (zona somera de la Caleta de Cuastecomate y Punta Corrales), áreas de fondos blandos (centro de la Caleta de
Cuastecomates y Melaque), y fondos mixtos arena-roca (Rojo-Vázquez et al. 2001). Esta bahía sostiene un intercambio continuo de agua con la Laguna de Barra de Navidad y se destacan algunas estructuras arrecifales de coral localizadas en las zonas semiprotegidas del litoral rocoso en la caleta de Cuastecomate.

La Bahía de Chamela se extiende a lo largo de $7.5 \mathrm{~km}^{2}$ aproximadamente, donde se encuentran grandes acantilados, zonas rocosas, playas arenosas de extensiones considerables (5-7 $\mathrm{km}^{2}$ ), y está protegida por más de una docena de islas e islotes. Los hábitats principales de esta bahía son el litoral rocoso con una notable abundancia y diversidad de macroalgas (Flores \& González 1981), zonas de bajos con arrecife de coral, playas de arena y pequeños estuarios que hacen aportes en años de buena precipitación. La configuración del sistema insular y los procesos costeros en la Bahía de Chamela, podrían constituir un sistema permanente de surgencias (Suárez-Morales et al.2000) muy localizado, que le confiere una singularidad ecológica a esta área.

Se recolectaron los estómagos de las 6 especies de peces más abundantes en la pesquería artesanal ribereña de la costa sur del estado de Jalisco; Caranx caballus (Günther1868), Haemulon flaviguttatum (Gill 1862), Microlepidotus brevipinnis (Steindachner 1869), Lutjanus argentiventris (Peters 1869), L. guttatus (Steindachner 1869), y Mulloidichthys dentatus (Gill 1862). Estas especies representan aproximadamente el $44 \%$ de la abundancia de las capturas totales de los últimos años (Rojo et al. 2008). Los estómagos de los ejemplares se obtuvieron de las capturas realizadas en Bahía de Navidad y Bahía de Chamela, en la costa sur del estado de Jalisco, utilizando redes de enmalle de cuatro tamaños diferentes de luz de malla: 7.6, 8.9, 10.1 , y $11.4 \mathrm{~cm}$; de aproximadamente $100 \mathrm{~m}$ de longitud y $4.5 \mathrm{~m}$ de altura.

De Junio de 2007 a Mayo de 2008 y durante una semana de cada mes, se realizaron actividades de pesca durante la noche, las redes se colocaron para operar en el fondo de manera perpendicular a la línea de costa en un rango de 7 a $12 \mathrm{~m}$ de profundidad. 
A todos los organismos capturados se les extrajo el contenido estomacal, que fue analizado en fresco en el laboratorio con la ayuda de un microscopio estereoscópico. Los organismos encontrados dentro de cada estómago se identificaron hasta el mínimo taxón posible con la ayuda de claves especializadas para cada grupo: Keen (1971), Brusca (1980), Allen y Robertson (1994), Fischer et al. (1995), Hendrickx (1996), Hendrickx y Estrada (1996), Hendrickx (1997a, b) y Salazar-Vallejo (1989).

Los componentes alimentarios de los estómagos analizados se cuantificaron de acuerdo con los métodos Numérico $\% N=$ $N_{i} / N_{t}$, donde $\mathrm{N}_{\mathrm{i}}$ es el número de organismos encontrados de una categoría $i$ y $N_{t}$ es el número total de organismos encontrados de todas las categorías. Gravimétrico $\% W=W_{i} /$ $W_{t}$, donde $W_{i}$ es el peso de la categoría $i$ y $W_{t}$ es el peso total de todas las categorias encontradas. Frecuencia de Ocurrencia $\% F O=N_{o} /$ $N_{s}$, donde $N_{o}$ es el número de ocurrencia de la categoría $i$ y $N_{s}$ es el número total de estómagos analizados (Hyslop 1980). La combinación de los tres índices anteriores da como resultado el Índice de Importancia Relativa (IIR) (Pinkas et al.1971) que permite valorar la importancia de cada tipo de alimento. Se utiliza para el análisis, evaluación y caracterización de los espectros tróficos de las especies estudiadas y se calcula con la siguiente fórmula:

$$
\mathrm{IIR}=(\mathrm{N} \%+\mathrm{W} \%)^{*} \mathrm{FO} \%
$$

Donde N\% es el porcentaje numérico, W\% porcentaje gravimétrico y FO\% es el porcentaje de frecuencia de ocurrencia de las presas encontradas. La evaluación de la amplitud del espectro trófico (amplitud del subnicho trófico) permite evaluar el nivel de especialización de los organismos, y se determinó mediante el índice de Levins (1968), el cual propone que la amplitud puede ser estimada a partir de la uniformidad de la distribución de los individuos entre los diversos recursos alimenticios (Krebs 1999),

$$
B=\frac{1}{\sum P_{j}^{2}}
$$

Donde $P j$ es la proporción con la cual cada categoría de la presa j contribuye a la dieta. Los valores obtenidos fueron estandarizados con el método de Hurlbert (1978) para ser expresados en una escala de 0 a 1.0 con la siguiente formula:

$$
B_{A}=\frac{B-1}{n-1}
$$

Donde $B A$ es máxima cuando la especie consume los diferentes recursos alimenticios en la misma proporción, lo que significa que la especie no discrimina entre los recursos alimenticios y por lo tanto su nicho trófico es el más amplio posible. Por el contrario, $B A$ alcanza su mínimo valor cuando los individuos se alimentan preferentemente de un único tipo de alimento (mínima amplitud de la dieta, máxima especialización). Para el traslape de dietas se utilizó el Índice Simplificado de Morisita propuesto por Horn (1966), el cual es un índice de similitud que puede ser usado para medir el traslape de nicho, y se calcula con la formula (Krebs 1999):

$$
C_{H}=\frac{2 \sum_{i}^{n} P_{i j} P_{i k}}{\sum_{i}^{n} P_{i j}^{2}+\sum_{i}^{n} P_{i k}^{2}}
$$

Donde $C_{H}$ es el Índice Simplificado de Morisita entre las especie $j$ y $k$; $P i j$ es la proporción del recurso $i$ del total de recursos utilizados por la especie $j ; P_{i k}$ es la proporción del recurso $i$ del total de recursos utilizados por la especie $k$; y $n$ es el número total de recursos utilizados. Según la escala propuesta por Langton (1982), los valores de este índice oscilan entre 0 y 1 . Valores cercanos a 0 indican ausencia de traslape en los componentes de la dieta. El valor de 1 indica un traslape completo. Valores mayores de 0.6 son indicadores de un traslape significativo, mientras que los valores menores de 0.29 indican un mínimo traslape en los componentes tróficos.

Los datos se agruparon para los análisis de acuerdo con las pautas hidroclimáticas registradas en la zona (Filonov et al. 2000, Franco-Gordo et al. 2001a, 2001b, 2003, 
Godínez-Domínguez \& Freire 2003), las cuales consideran dos periodos principales: influencia de la Contracorriente Norecuatorial (T1) (juliodiciembre), y periodo de influencia de la Corriente de California (T2) (enero-junio).

Para analizar gráficamente el patrón de asociación de presas de acuerdo a la especie de depredador, periodo hidroclimático y sitio de muestreo, se utilizó el método de escalamiento multidimensional no métrico (por sus siglas en inglés nMDS) (Clarke 1993), por medio del programa estadístico PRIMER v5.2.2 (Clarke \& Gorley 2004). Previamente los valores de abundancia de las dietas fueron transformados a raíz cuarta, y se estimaron los coeficientes de similitud de Bray Curtis (Clarke \& Warwick 2001).

\section{RESULTADOS}

Análisis de la dieta: Se recolectaron un total de 1183 estómagos de las seis especies de peces estudiadas en ambos sitios de muestreo. En Bahía de Navidad se analizaron 589 estómagos, y se identificaron 78 presas. En el periodo comprendido de julio a diciembre (T1) se identificaron 64 presas y 45 de enero a junio (T2). Para la Bahía de Chamela se identificaron 93 presas en 594 estómagos, se identificaron 74 presas en el periodo $\mathrm{T} 1$ y 60 presas para el periodo T2 (Cuadro 1).

Contenidos estomacales. Bahía de Navidad: C. caballus. En el periodo $\mathrm{T} 1$ se analizaron los estómagos de 21 organismos entre $23.7 \mathrm{~cm} \mathrm{y} 55.7 \mathrm{~cm}$ de longitud total (Lt), y se encontraron 17 presas. Los peces clupeidos fueron el componente de mayor importancia en la dieta con $94.2 \%$ del IIR. En el periodo T2 fueron examinados 90 organismos entre 22 y $47.5 \mathrm{~cm}$ y se encontraron 14 presas; el $92 \%$ de ellas estuvo representado por misidaceos (42.9\% IIR), peces clupeidos (33.5\% IIR), camarones de la familia Processidae (9.3\% IIR) y cefalópodos (5.9\% IIR) (Cuadro 2).

H. flaviguttatum. Durante T1 se identificaron siete presas en estómagos de 24 organismos con una Lt entre 17.3 y $38.7 \mathrm{~cm}$. Las larvas de

CUADRO 1

Número de estómagos y presas encontradas en las especies de peces analizadas por periodo y sitio de muestreo

TABLE 1

Number of stomachs and preys found in the fish species analyzed, by period and study site

\begin{tabular}{|c|c|c|c|c|}
\hline & \multicolumn{4}{|c|}{ Bahía de Navidad } \\
\hline & \multicolumn{2}{|c|}{$\mathrm{T} 1$} & \multicolumn{2}{|c|}{ T 2} \\
\hline & Estómagos analizados & Presas encontradas & Estómagos analizados & Presas encontradas \\
\hline C. caballus & 21 & 17 & 90 & 14 \\
\hline H. flaviguttatum & 24 & 7 & 15 & 10 \\
\hline M. brevipinnis & 89 & 22 & 50 & 18 \\
\hline M. dentatus & 60 & 35 & 52 & 34 \\
\hline L. argentiventris & 25 & 24 & 36 & 21 \\
\hline \multirow[t]{4}{*}{ L. guttatus } & 19 & 28 & 56 & 15 \\
\hline & \multicolumn{4}{|c|}{ Bahía de Chamela } \\
\hline & \multicolumn{2}{|c|}{$\mathrm{T} 1$} & \multicolumn{2}{|c|}{ Т 2} \\
\hline & Estómagos analizados & Presas encontradas & Estómagos analizados & Presas encontradas \\
\hline C. caballus & 14 & 22 & 58 & 10 \\
\hline H. flaviguttatum & 19 & 18 & 19 & 19 \\
\hline M. brevipinnis & 42 & 36 & 46 & 30 \\
\hline M. dentatus & 49 & 45 & 49 & 36 \\
\hline L. argentiventris & 41 & 28 & 37 & 28 \\
\hline L. guttatus & 62 & 13 & 14 & 30 \\
\hline
\end{tabular}




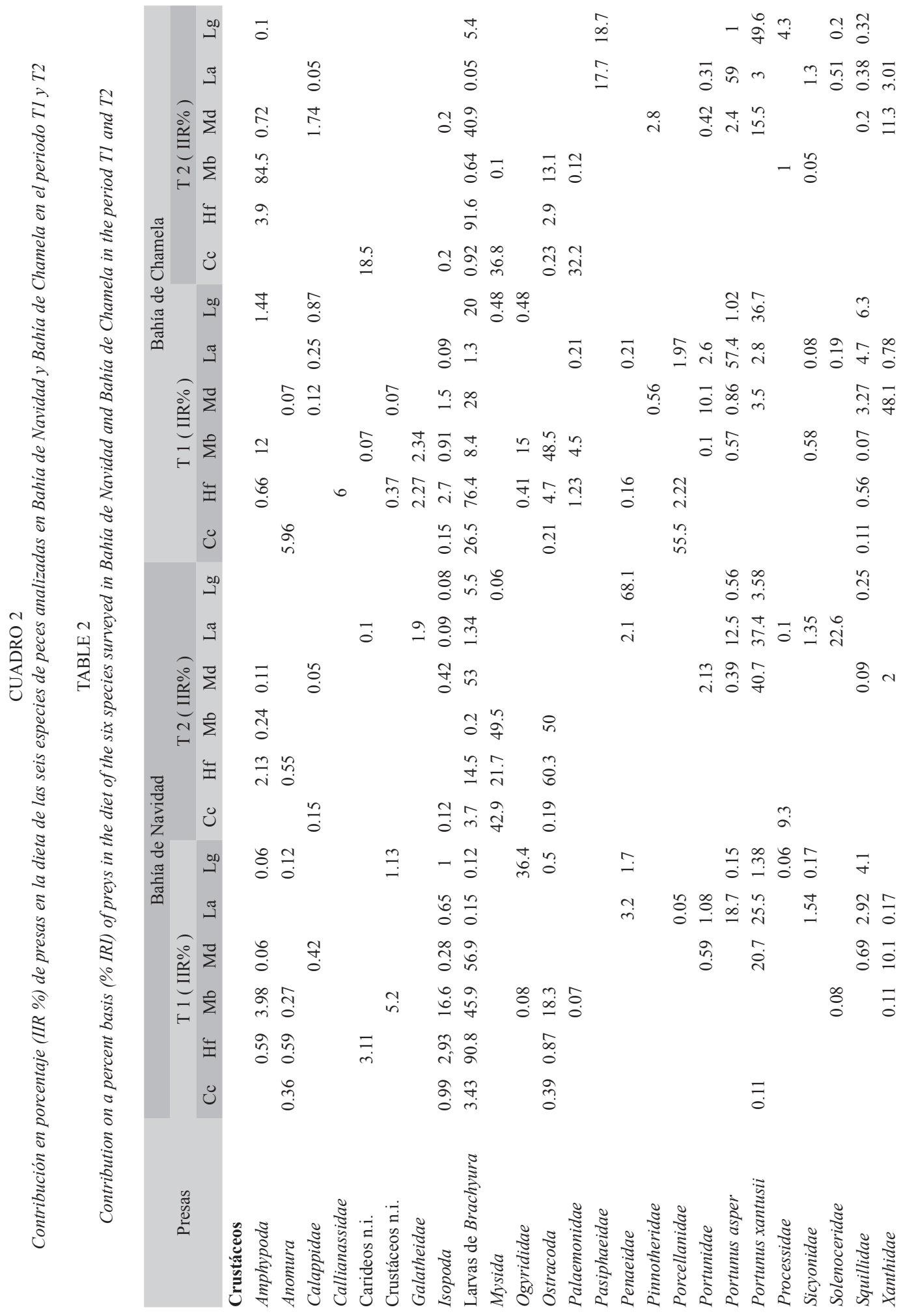




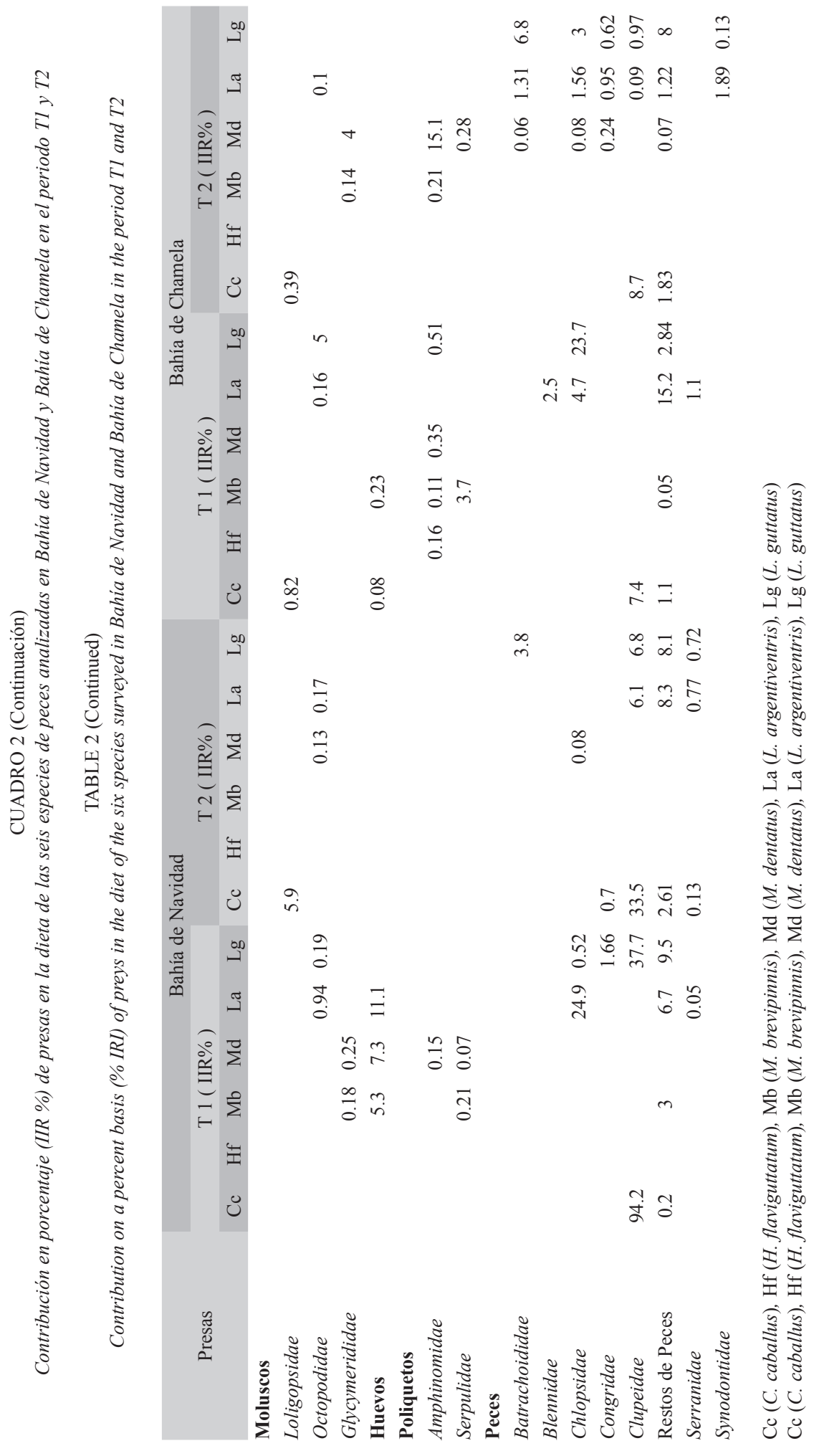


braquiuros no identificadas constituyeron el $90.8 \%$ del IIR de la dieta. En el periodo T2 se encontraron 10 presas en 15 organismos con una Lt entre los 22 y $35.5 \mathrm{~cm}$. El $90 \%$ del IIR de la dieta total estuvo formado por ostrácodos (60.3\% IIR), misídaceos (21.7\% IIR) y larvas de braquiuros (14.5\% IIR) (Cuadro 2).

M. brevipinnis. Durante el periodo $\mathrm{T} 1$ se analizaron los estómagos de 89 organismos entre 21 y $38.9 \mathrm{~cm}$, y la dieta estuvo compuesta por 22 presas. Las más importantes de acuerdo al IIR fueron las larvas de braquiuros (45.9\%), ostrácodos $(18.3 \%)$, isópodos $(16.6 \%)$, huevos no identificados $(5.3 \%)$, y restos de crustáceos (5.2\%), que acumularon más del 90\%. En el periodo T2 se analizaron estómagos de 50 organismos entre 19.5 y $38.2 \mathrm{~cm}$ de Lt y se encontraron 18 presas. Los ostrácodos $(50.0 \%$ IIR) y los misidáceos (49.5\%) comprendieron más del 99\% del IIR de la dieta (Cuadro 2).

$M$. dentatus. En el periodo T1 se identificaron 35 presas en 60 estómagos de organismos entre 25.4 y los $38.8 \mathrm{~cm}$ de Lt, de las cuales, cuatro presas completaron más del $90 \%$ del IIR de la dieta. Las más importantes fueron las larvas de braquiuros (56.9\% IIR), Portunus xantusii (20.7\% IIR), cangrejos de la familia Xanthidae (10.1\% IIR) y huevos n.i. (7.3\% IIR). En 52 organismos entre los 21.5 y $36.6 \mathrm{~cm}$ en el periodo $\mathrm{T} 2$ se identificaron 34 presas. Las larvas de braquiuro (53.0\% IIR) y $P$. xantusii (40.7\% IIR) alcanzaron casi el 94\% del IIR de la dieta (Cuadro 2).

L. argentiventris. Se encontraron 24 presas en el periodo T1 en 25 organismos entre los 27.8 y $58.6 \mathrm{~cm}$ de Lt. P. xantusii (25.5\% IIR), peces de la familia Chlopsidae (24.9\% IIR), Portunus asper (18.7\% IIR), huevos n.i. (11.1\% IIR), los restos de peces n.i. (6.7\% IIR) y camarones de la familia Penaeidae (3.2\% IIR) acumularon aproximadamente el $90 \%$ de la dieta. En T2 se identificaron 21 presas en 36 organismos analizados entre los $23.4 \mathrm{~cm}$ y $62 \mathrm{~cm}$ de $\mathrm{Lt}$, y de estas presas la de mayor importancia fue P. xantusii con el $37.4 \%$ del IIR, seguido por camarones de la familia Solenoceridae $(22.6 \%$ IIR), $P$. asper (12.5\% IIR), peces n.i. (8.3\% IIR), peces clupeidos (6.1\% IIR), camarones peneidos (2.1\% IIR) y langostillas de la familia Galatheidae (1.9\% IIR) (Cuadro 2).

L. guttatus. En 19 organismos analizados con una Lt entre 24 y $51.6 \mathrm{~cm}$, durante el periodo $\mathrm{T} 1$ consumieron 28 presas, de las cuales cuatro representaron el $90 \%$ del total de la dieta. Las más importantes fueron los peces clupeidos $(37.7 \%)$, camarones carideos de la familia Ogyrididae (36.4\% IIR), restos de peces n.i. (9.5\% IIR), estomatópodos (4.1\%) y camarones peneidos (1.7\% IIR). En T2 se analizaron 56 organismos entre 17.7 y $44 \mathrm{~cm}$ de Lt se encontraron 15 presas, de las cuales los camarones peneidos son los de mayor importancia en la dieta (68.1\% IIR), seguidos de peces n.i. (8.1\% IIR), peces clupeidos (6.8\% IIR), larvas de braquiuros (5.5\% IIR), y Porichthys margaritatus $(3.8 \%$ IIR) que alcanzaron más del 90\% del IIR de la dieta (Cuadro 2).

Bahía de Chamela. C. caballus. En el periodo $\mathrm{T} 1$ se analizaron 14 organismos entre 34 y $43.4 \mathrm{~cm}$ de Lt y se encontraron 22 presas. El 90\% del IIR de la dieta está compuesta por larvas del género Porcellana (55.5\%), larvas de braquiuros $(26.5 \%)$, peces clupeidos $(7.4 \%)$ y restos de peces n.i. (1.1\%), que juntas alcanzan el $90 \%$ total de las presas consumidas. En T2 se identificaron únicamente 10 presas en 58 organismos con una Lt entre 21.6 y 47.8 $\mathrm{cm}$, y cuatro de ellas sobrepasan el $90 \%$ de la dieta; misidaceos (36.8\% IIR), camarones de la familia Palaemonidae (32.2\% IIR), camarones carideos n.i. (18.5\% IIR) y peces clupeidos (8.7\% IIR) (Cuadro 2).

H. flaviguttatum. Se analizaron 19 organismos entre 22 y $41.2 \mathrm{~cm} \mathrm{Lt}$ en ambos periodo de estudio. En $T 1$, consumieron 18 presas. El $76.4 \%$ IIR de la dieta fueron larvas de braquiuros, macruros de la familia Callianassidae $(6 \%)$, ostrácodos $(4.7 \%)$, e isópodos $(2.7 \%)$, que representaron el $90 \%$ del IIR. En T2 se encontraron 19 presas, de las cuales tres microcrustáceos; larvas de braquiuros (91.6\% IIR), anfípodos (3.9\% IIR) y ostrácodos (2.9\% IIR) acumularon la mayor proporción de la dieta, y las 15 presas restantes alcanzaron el $1.6 \%$ del IIR en este periodo (Cuadro 2). 
M. brevipinnis. Se analizaron 42 estómagos de organismos entre 25 y $37.2 \mathrm{~cm}$ en el periodo $\mathrm{T} 1$, y se encontraron 36 presas. La dieta estuvo compuesta por ostrácodos $(48.5 \%$ IIR), camarones de la familia Ogyrididae (15\% IIR), anfípodos (12\% IIR), larvas de braquiuros (8.4\% IIR), camarones palemónidos $(4.5 \%$ IIR), poliquetos de la familia Serpulidae (3.7\% IIR) y otras 30 presas más (7.5\% IIR). En el periodo $\mathrm{T} 2$ se analizaron 46 organismos entre 23.6 y $40.7 \mathrm{~cm}$ de Lt y se encontró que son dos los principales componentes en la dieta, anfípodos (84.5\%) y ostrácodos (13.1\% IIR). El resto de la dieta se completa con otras 28 presas (Cuadro 2).

M. dentatus. Se examinaron 49 organismos para cada periodo con Lt entre 22.5 y $37.2 \mathrm{~cm}$. En el periodo T1 tuvo el mayor número de presas (45 presas) consumidas de todas las especies en ambas temporadas y sitios, de las cuales, cinco representan más del $90 \%$ de la dieta. Las presas de mayor importancia son los cangrejos de la familia Xanthidae (48.1\% IIR), las larvas de braquiuros (28\% IIR), los cangrejos portúnidos n.i. (10.1\% IIR), P. xantusii (3.5 $\%$ IIR) y los isópodos (1.5\% IIR). En T2 consumió 36 presas, y el 90\% del IIR de la dieta esta compuesto por larvas de braquiuros (40.9\% IIR), P. xantusii (15.5\% IIR), poliquetos de la familia Amphinomidae (15.1\% IIR), cangrejos de la familia Xanthidae (11.3\% IIR), pequeños bivalvos de la familia Glycymeridae (4\% IIR), cangrejos de la familia Pinnotheridae $(2.8 \%$ IIR) y P. asper (2.4\% IIR) (Cuadro 2).

L. argentiventris. Se identificaron 28 presas en 41 organismos analizados con Lt entre 23.8 y $57.2 \mathrm{~cm}$ en el periodo T1. El $90 \%$ de la dieta estuvo compuesta por siete presas, P. asper (57.4\% IIR), peces n.i. (15.2\% IIR), peces de la familia Chlopsidae (4.7\% IIR), estomatópodos (4.7\% IIR), P. xantusii $(2.8 \%$ IIR), otros cangrejos portúnidos (2.6\% IIR) y peces de la familia Blennidae (2.5\% IIR). En T2 se analizaron 37 organismos entre 25.2 y $43.5 \mathrm{~cm}$ de Lt y consumieron 28 presas, y como en T1 $P$. asper alcanzó la mayor importancia (59\% IIR), seguida por camarones peneidos (17.7\% IIR), P. xantusii (3\%) y cinco familias de peces (5.8\% IIR) completaron más del 90\% del IIR de la dieta (Cuadro 2).

L. guttatus. En el periodo T1 se identificaron 13 presas en 62 organismos analizados entre los 21.7-57.2cm de Lt, de las cuales cinco sobrepasan del $90 \%$ del IIR de la dieta. El principal componte alimenticio fue $P$. xantusii (36.7\% IIR), seguido por peces de la familia Chlopsidae (23.7\% IIR), las larvas de braquiuros (20\% IIR), estomatópodos (6.3\% IIR) y el pulpo Octopus hubssorum (5\% IIR). En el periodo T2 se analizaron 14 organismos con una Lt entre los $24.9-40.5 \mathrm{~cm}$ y se encontraron 30 presas; la más abundante fue $P$. xantusii $(49.6 \%)$, además de camarones peneidos (18.7\% IIR), peces n.i. (7.9\% IIR), P. margaritatus $(6.86 \%$ IIR), larvas de braquiuros (5.4\% IIR) y camarones carideos de la familia Processidae (4.3\% IIR), alcanzan más del 90\% IIR de la dieta consumida (Cuadro 2).

\section{Amplitud y superposición de subnichos} tróficos. Los valores del índice de amplitud de nicho de Levins indican una estrategia especialista en la dieta de todas las especies, ya que en todos los casos están por debajo de 0.6 , en ambos periodos y sitios de muestreo (Cuadro 3).

El índice simplificado de Morisita (Horn 1966) muestra en general valores de traslape

CUADRO 3

Valores del Índice de Levins de las seis especies de peces en las dos localidades y periodos de muestreo

TABLE 3

Levins's index values of the six fish species in both localities and sampling periods

\begin{tabular}{l|cccc} 
& \multicolumn{2}{c}{$\begin{array}{c}\text { Bahía de } \\
\text { Navidad }\end{array}$} & \multicolumn{2}{c}{$\begin{array}{c}\text { Bahía de } \\
\text { Chamela }\end{array}$} \\
\cline { 2 - 5 } & T1 & T2 & $T 1$ & T2 \\
\hline C. caballus & 0.01 & 0.17 & 0.07 & 0.28 \\
H. flaviguttatum & 0.04 & 0.15 & 0.04 & 0.01 \\
M. brevipinnis & 0.12 & 0.06 & 0.07 & 0.01 \\
M. dentatus & 0.05 & 0.04 & 0.04 & 0.09 \\
L. argentiventris & 0.20 & 0.18 & 0.07 & 0.06 \\
L. guttatus & 0.09 & 0.07 & 0.27 & 0.08
\end{tabular}


de dietas bajos entre las especies, sitios y periodos. En la Bahía de Navidad en el periodo T1, $H$. flaviguttatum, M. dentatus y M. brevipinnis presentaron un traslape significativo (mayor a 0.77 ), y L. guttatus comparte un valor de 0.6 de la dieta con C. caballus. Durante el periodo T2 H. flaviguttatum y $M$. brevipinnis presentaron un traslape importante (0.82). En Bahía de Chamela, el número de traslapes de dietas significativos fueron aún menos; sólo H. flaviguttatum y $M$. dentatus presentaron un traslape de dieta significativo $(0.71)$ durante T2 (Cuadro 4).

El escalamiento multidimensional no métrico (Fig. 1) indica que sólo las especies de depredadores contribuyen a explicar el patrón de asociación de presas, mientras que factores como periodo hidroclimático y sitio de muestreo, tienen una contribución mínima. De acuerdo a la similitud de las asociaciones de presas, los depredadores se agrupan de la siguiente manera: un grupo formado por las especies del

CUADRO 4

Valores del traslape de dietas estimadas por el indice Simplificado de Morisita de las seis especies de peces en ambas localidades y estaciones hidroclimáticas

TABLE 4

Values of the niche overlap estimated by the Simplified Morisita Index of the six species at both stations and hydroclimatic periods

\begin{tabular}{lccccc}
\multicolumn{7}{c}{ Bahía de Navidad } \\
\multicolumn{1}{c}{ T1 } & H. flaviguttatum & M. brevipinnis & M. dentatus & L. argentiventris & L. guttatus \\
C. caballus & 0.40 & 0.03 & 0.03 & 0.01 & 0.60 \\
H. flaviguttatum & & 0.77 & 0.86 & 0.01 & 0.01 \\
M. brevipinnis & & 0.80 & 0.04 & 0.02 \\
M. dentatus & & & 0.22 & 0.01 \\
L. argentiventris & & & & 0.06
\end{tabular}

\begin{tabular}{|c|c|c|c|c|c|}
\hline $\mathrm{T} 2$ & H. flaviguttatum & M. brevipinnis & M. dentatus & L. argentiventris & L. guttatus \\
\hline C. caballus & 0.27 & 0.53 & 0.05 & 0.09 & 0.07 \\
\hline H. flaviguttatum & & 0.82 & 0.17 & 0.01 & 0.02 \\
\hline M. brevipinnis & & & 0.01 & 0.01 & 0.01 \\
\hline M. dentatus & & & & 0.48 & 0.09 \\
\hline L. argentiventris & & & & & 0.11 \\
\hline
\end{tabular}

\begin{tabular}{lccccc}
\multicolumn{1}{c}{ Bahía de Chamela } \\
\multicolumn{1}{c}{ T1 } & H. flaviguttatum & M. brevipinnis & M. dentatus & L. argentiventris & L. guttatus \\
C. caballus & 0.44 & 0.07 & 0.21 & 0.04 & 0.17 \\
H. flaviguttatum & & 0.20 & 0.47 & 0.02 & 0.37 \\
M. brevipinnis & & 0.08 & 0.01 & 0.07 \\
M. dentatus & & & 0.05 & 0.25 \\
L. argentiventris & & & & 0.13
\end{tabular}

\begin{tabular}{lccccc}
\multicolumn{1}{c}{ T2 } & H. flaviguttatum & M. brevipinnis & M. dentatus & L. argentiventris & L. guttatus \\
C. caballus & 0.02 & 0.01 & 0.01 & 0.01 & 0.01 \\
H. flaviguttatum & & 0.05 & 0.71 & 0.01 & 0.09 \\
M. brevipinnis & & 0.02 & 0.01 & 0.01 \\
M. dentatus & & & 0.09 & 0.38 \\
L. argentiventris & & & & 0.22
\end{tabular}




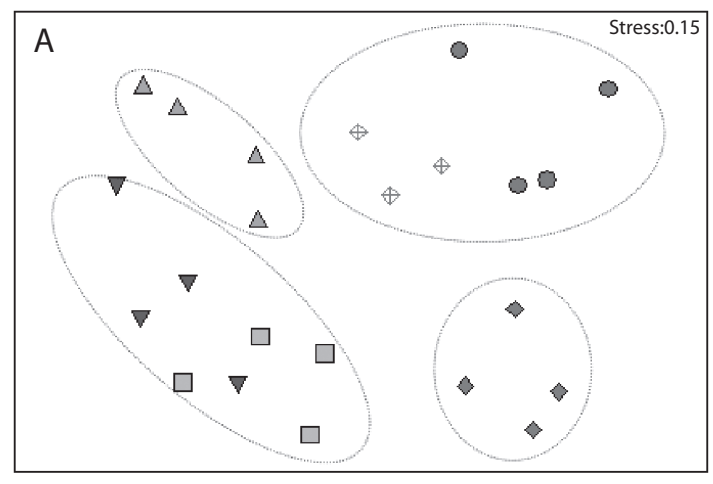

$\triangle$ C. caballus

$\nabla$ H. flaviguttatum

$\square$ M. brevipinnis

M. dentatus

L. argentiventris

$\uparrow$ L. guttatus

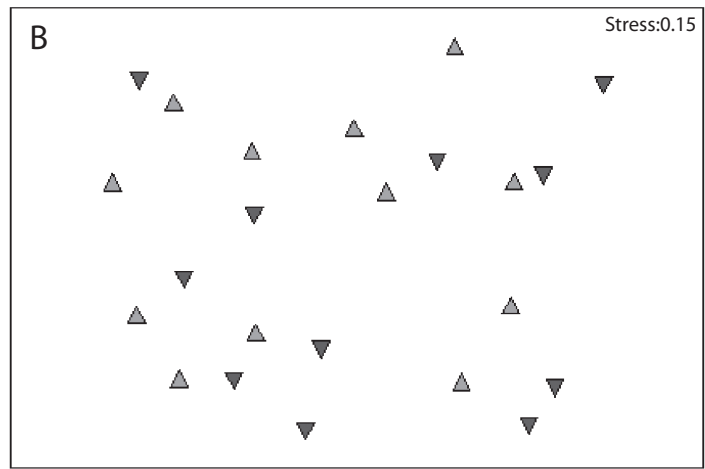

$\triangle$ Navidad

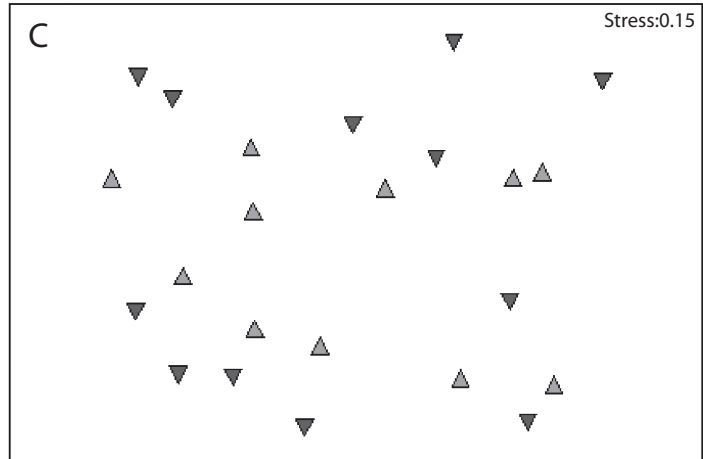

Chamela

$\triangle T 1$

Fig. 1. Patrón de similitud de las asociaciones de especies de presas obtenido con el escalamiento multidimensional no métrico, en función de A) peces depredadores B) sitios de muestreo C) periodo hidroclimático.

Fig. 1. Similarity pattern of the prey items assemblages determined with non-metric multidimensional scaling (nMDS) according to A) predator fishes B) samplings sites C) hydroclimatic period.

género Lutjanus (guttatus y argentiventris), el segundo grupo formado por las especies de la familia Haemulidae (H. falviguttatum y $M$. Brevipinnis), y dos grupos uniespecíficos formados C. caballus y M. dentatus.

\section{DISCUSIÓN}

El análisis de los componentes alimenticios en las seis especies de peces de mayores abundancias de captura en Bahía de Navidad 
y Chamela en la pesca ribereña, muestra que su dieta está constituida principalmente por crustáceos (57-78\%), peces $(27 \%)$ y moluscos (5-15\%). Otras entidades alimentarias frecuentes fueron huevos no identificados, poliquetos y equinodermos en menores proporciones.

La riqueza de presas encontradas en los estómagos de las seis especies de peces estudiadas en ambos periodos, fue mayor en la Bahía de Chamela, con excepción de C. caballus en T2 y L. guttatus en T1. Esto puede ser debido probablemente a que existe una mayor abundancia y riqueza de macroinvertebrados bentónicos de fondos blandos en Bahía de Chamela (Godínez-Domínguez et al. com. pers.), y una mayor biomasa zooplanctónica (FrancoGordo et al. com. pers.).

La alimentación de C. caballus está basada en organismos pelágicos, como peces clupeidos e invertebrados del zooplancton, como lo han reportado en otras especies de este género (Meyer et al. 2001). Sin embargo, FloresOrtega (2004) observó, a diferencia de lo anterior, una dieta para esta especie en la Bahía de Navidad compuesta en un $85 \%$ por macroinvertebrados bentónicos de fondos blandos (camarones penaeidos, cangrejos portúnidos y estomatópodos), con peces y cefalópodos en menores proporciones. Bernal-Ornelas (2007) en la Bahía de Manzanillo observó variaciones en la selectividad del alimento conforme a la talla de los organismos analizados, sin embargo, su principal componente fueron peces y crustáceos bentónicos.

L. argentiventris y L. guttatus son considerados grandes depredadores con hábitos variados de alimentación. Estas dos especies tienen preferencia por los crustáceos bentónicos como cangrejos portúnidos ( $P$. asper, $P$. xantusii), camarones (Familia Penaeidae y Solenoceridae) y peces (Fam. Chlopsidae y Clupeidae) en menor cantidad durante todo el año. Sin embargo, la preferencia entre peces y crustáceos puede variar entre años (Saucedo-Lozano \& Chiappa-Carrara 2000). Estos autores encontraron para la misma área de estudio de este trabajo, una dieta de L. guttatus compuesta principalmente por peces (Fam. Congridae y Eucinostomus gracilis) y crustáceos (Fam. Penaeidae), alternando a lo largo del año una dominancia de ciertas presas. La diversidad y preferencia por algunas presas está documentada en México por Santamaría-Miranda et al. (2005), quienes encontraron en la dieta de L. argentiventris al norte de Sinaloa que las principales presas son crustáceos bentónicos (63\%) así como cangrejos y camarones, y peces (37\%), mientras que Vázquez et al. (2008) en la Bahía de La Paz, reportaron que esta especie se alimenta principalmente de peces (95\%) y en menor cantidad de crustáceos (2\%). Por lo tanto, estas especies tienen la capacidad de alimentarse tanto en la zona pelágica como en los fondos blandos o rocosos, lo cual es una característica distintiva de la familia Lutjanidae (Fisher et al. 1995).

H. flaviguttatum y M. brevipinnis, son peces cuya dieta está compuesta en más del $90 \%$ por invertebrados pequeños que se encuentran como parte del plancton o en fondos blandos y rocosos, lo cual concuerda con los resultados obtenidos por Morales-Jauregui (2007) y Flores-Ortega (2004) para estas especies respectivamente en la Bahía de Navidad, y Raymundo (2000) para H. maculicauda en la costa de Jalisco y Colima, México.

En las dos bahías estudiadas no se tienen antecedentes de estudios sobre aspectos alimenticios de $M$. dentatus, Raymundo-Huizar (2000) estudió la alimentación de una especie de esta misma familia, Pseudupeneus grandisquamis y obtuvo una dieta compuesta principalmente por crustáceos y en segundo término por poliquetos y peces. Tanto en Chamela como en Bahía de Navidad, los crustáceos fueron el componente más importante de la dieta de $M$. dentatus, y sólo en Chamela durante T2 los poliquetos representaron el 15\% IIR.

Estas seis especies estudiadas comparten un hábitat costero marino, pero ésta no ha sido una condición que las obligue a consumir las mismas presas. Las diferencias en la composición de la dieta pueden estar determinadas por distintos factores, tales como el tamaño de la boca, ya que la apertura de la boca define el tamaño máximo de las presas que consume 
una especie, y los hábitats donde se alimentan (Gerking 1994). Este es el caso de M. brevipinnis, H. flaviguttatum y $M$. dentatus que son peces de boca pequeña, por lo que sólo pueden consumir organismos pequeños y con exosqueletos delgados, ya que no cuentan con una dentadura fuerte como la de C. caballus, $L$. argentiventris y L. guttatus. Estas últimas están adaptadas para consumir presas con estructuras óseas como peces y exoesqueletos duros como los cangrejos braquiuros, algunos equinodermos y moluscos pequeños.

Las estrategias que emplean los peces para alimentarse están influenciadas por altas y bajas en la abundancia de las presas o en la diversidad de los recursos alimenticios (Pratchett \& Berumen 2008). En el periodo $\mathrm{T} 1$ en las dos bahías se observó el mayor número de presas consumidas por las seis especies analizadas. Sin embargo, este incremento en el número de presas encontradas, no corresponde con la riqueza de macroinvertebrados de fondos blandos encontrada por Godínez-Domínguez et al. (2009), donde no se observaron diferencias en la riqueza de especies entre periodos. En un estudio reciente de los macroinvertebrados de la infauna de fondos blandos someros de la Bahía de Navidad (Corgos et al. com. pers.), tampoco se observaron diferencias en la riqueza de especies entre periodos hidroclimáticos. Mientras que en relación a la disponibilidad de las presas, Godínez-Domínguez \& González-Sansón (1998) observaron en el periodo T1, una menor abundancia de macroinvertebrados epibentónicos, Corgos et al. (com. pers.), observaron las mayores abundancias de macroinvertebrados infaunales en este periodo.

Las seis especies aquí estudiadas, muestran una apropiada partición de los recursos existentes en ambas bahías. El gran nivel de especialización de las dietas y los bajos valores en general de los traslapes en las dietas, pueden reforzar la idea de que no existen indicadores de vulnerabilidad provocados por la competencia entre las especies cuando la disponibilidad y la abundancia de los recursos disminuyen por factores espaciales o temporales (Bulman et al. 2002).

\section{AGRADECIMIENTOS}

El presente trabajo fue financiado por la Universidad de Guadalajara y el Programa de Mejoramiento del Profesorado (SEP-PROMEP) y el apoyo del Cuerpo Académico Consolidado Ecología y Recursos Marinos (UDG-CA-494). Los autores agradecen a la Sociedad Cooperativa de Producción Pesquera Punta Farallón y a la SCPP Ribera de Melaque por el apoyo en la operación de las redes de pesca, en especial a Manuel -el güero- y Tino. A Gabriela Lucano Ramírez, Salvador Ruíz Ramírez, y Yael Morales Jauregui que apoyaron en la extracción del material biológico.

\section{RESUMEN}

Se analizaron los componentes tróficos de las seis especies de peces más importantes en la captura de la pesquería artesanal en las Bahía de Navidad y Chamela, ubicadas en la costa de Jalisco, México. Las especies estudiadas fueron Microlepidotus brevipinnis, Caranx caballus, Haemulon flaviguttatum, Lutjanus guttatus, Lutjanus argentiventris y Mulloidichthys dentatus. Se consideraron dos periodos de estudio de acuerdo a las pautas hidroclimáticas y los patrones de producción biológica en la zona: periodo de influencia de la Contracorriente Norecuatorial (T1), y periodo de influencia de la Corriente de California (T2). En Bahía de Navidad se identificaron 78 presas en total para las seis especies. En el periodo comprendido entre julio y diciembre (T1) se identificaron 64 presas y 45 de enero a junio (T2). En Bahía de Chamela se identificaron 93 presas, encontrando 74 presas en el periodo T1 y 60 presas para el periodo T2. El mayor número de presas se encontró en $M$. dentatus con 34 presas en el periodo T1 de Bahía de Chamela y el menor número fue en $H$. flaviguttatum en T1 de Bahía de Navidad con solo 7 presas. Los crustáceos fueron las presas de mayor presencia en la dieta, seguidos por peces, moluscos, poliquetos y equinodermos, en ambos periodos y sitios de muestreo. Las seis especies analizadas se consideran como especialistas, dados los bajos valores del índice de amplitud de nicho en ambos periodos y sitios de muestreo.

Palabras clave: ecología trófica, peces tropicales, patrones hidroclimáticos, amplitud de nicho, partición de hábitat.

\section{REFERENCIAS}

Allen, G.R. \& D.R. Robertson. 1994. Fishes of the tropical eastern pacific. University of Hawaii, Hawaii, EEUU. 
Bernal-Ornelas, I.H. 2007. Alimentación del cocinero Caranx caballus (Pisces:Carangidae) Günther, 1868, en la costa de Manzanillo, Colima. Tesis de Licenciatura. Universidad de Guadalajara, Autlan de Navarro, Jalisco, México.

Berumen, M.L., M.S. Pratchett \& M.I. McCormick. 2005. Within-reef differences in diet and body condition of coral communities at Tiahura Reef, Moorea. Coral Reefs 25: 647-653.

Blaber, S.J.M. 1997. Fish and fisheries of tropical estuaries. Chapman \& Hall. Londres, Inglaterra.

Brusca, R.C.1980. Common intertidal invertebrates of the Gulf of California. University of Arizona, Tucson, Arizona, EEUU

Bulman, C.M., X. He \& J.A. Koslow. 2002. Trophic ecology of the mid-slope demersal fish community off southern Tasmania, Australia. Mar. Freshw. Res. 53: $59-72$.

Clarke, K.R. 1993. Non-parametric multivariate analyses of changes in community structure. Aust. J. Ecol. 18: $117-143$

Clarke, K.R. \& R.M. Warwick. 2001. Change in marine communities: an approach to statistical analysis and interpretation. PRIMER-E, Plymouth Laboratory, UK.

Clarke, K.R. \& R.N. Gorley. 2004. PRIMER v5: User Manual/Tutorial. PRIMER-E, Plymouth Laboratory, UK.

Edgar, G.J. \& C. Shaw. 1995a. The production and trophic ecology of shallow-water fish assemblages in southern Australia. II. Diets of fishes and trophic relationships between fishes and benthos at Western Port Victoria. J. Exp. Mar. Biol. Ecol. 194: 83-106.

Edgar, G.J. \& C. Shaw, 1995b. The production and trophic ecology of shallow-water fish assemblages in southern Australia. III. General relationships between sediments, seagrasess, invertebrates and fishes. J. Exp. Mar. Biol. Ecol. 194: 107-131.

Filonov, A.E., I.E. Tereshchenko, C.O. Monzón, M.E. González-Ruelas \& E. Godínez-Domínguez. 2000. Variabilidad estacional de los campos de temperatura y salinidad en la zona costera de los estados de Jalisco y Colima, México. Cienc. Mar. 26: 303-321.

Fischer, W., F. Krupp, W. Scheinder, C. Sommer, K. E. Carpenter \& V. H. Niem (Eds.) 1995. Pacífico Centro-Oriental. Guía FAO para la identificación de especies para los fines de la pesca. Vol. I-II-III. FAO, Roma, Italia.
Flores-Ortega, J.R. 2004. Componentes tróficos de algunas especies de peces de importancia comercial de Bahía de Navidad, Jalisco, México. Tesis Licenciatura, Instituto Tecnológico de Mar No. 6, Bahía de Banderas, Nayarit, México.

Flores-Pedroche, F. \& J. González-González, 1981. Lista preliminar de las algas marinas de la región sur de la costa de Jalisco, México. Phycol. Latinoam. 1: 60-72.

Franco-Gordo, C., E. Godínez-Domínguez \& E. SuárezMorales. 2003. Zooplancton diversity in the central Mexican Pacific. Estuar. Coast. Shelf Sci. 57: 111121.

Franco-Gordo, C., E. Godínez-Domínguez \& E. SuárezMorales. 2001. Zooplancton Biomass variability in the Mexican Eastern tropical Pacific. Pac. Sci. 55: 191-202.

Franco-Gordo, C., E. Suárez-Morales, E. Godínez-Domínguez \& R. Flores-Vargas. 2001. A seasonal survey of the fish larvae community of the central Pacific coast of México. B. Mar. Sci. 68: 383-396.

Gerking, S. D. 1994. Feeding Ecology of Fish. Academia, Nueva York, Nueva York, EEUU.

Godínez-Domínguez, E. \& J. Freire. 2003. An informationtheoretic approach for selection of spatial and temporal models of community organization. Mar. Ecol. Prog. Ser. 253: 17-24.

Godínez-Domínguez, E. \& G. González-Sansón. 1998. Variación de los patrones de distribución batimétrica de la fauna macrobentónica en la plataforma continental de Jalisco y Colima, México. Cienc. Mar. 24: 337-351.

Godínez-Domínguez, E., J. Freire, C. Franco-Gordo \& G. González-Sansón. 2009. Decomposing diversity patterns of a soft-bottom macroinvertebrate community in the tropical eastern Pacific. J. Mar. Biol. Assoc. U.K. 89: 31-38.

Granado-Lorencio, C., 2002. Ecología de peces. Universidad de Sevilla, Sevilla, España.

Helfman, G.S., J.L. Meyer \& W.N. MacFarland. 1982. The ontogeny of twilight migration patterns in grunts (Pisces: Haemulidae). Anim. Behav. 30: 317-326.

Hendrickx, M.E. 1996. Los camarones Penaeoidea Bentónicos (Crustacea: Decapoda: Dendrobranchiata) del Pacífico Mexicano. CONABIO. Instituto de Ciencias del Mar y Limnología. Universidad Nacional Autónoma de México, México. 
Hendrickx, M.E. 1997a. Los cangrejos Braquiuros (Crustacea: Brachyura: Dromiidae hasta Leucosiidae) del Pacífico Mexicano. CONABIO. Instituto de Ciencias del Mar y Limnología. Universidad Nacional Autónoma de México, México.

Hendrickx, M.E. 1997b. Los cangrejos Braquiuros (Crustacea: Brachyura: Majoidea y Parthenopoidea) del Pacífico Mexicano. CONABIO. Instituto de Ciencias del Mar y Limnología. Universidad Nacional Autónoma de México, México.

Hendrickx, M.E. \& F.D. Estrada-Navarrete. 1996. Los camarones Pelágicos (Crustacea: Decapoda: Dendrobranchiata) del Pacífico Mexicano CONABIO. Instituto de Ciencias del Mar y Limnología. Universidad Nacional Autónoma de México, México.

Horn, H.S. 1966. Measurement of "overlap" in comparative ecological studies. Am. Nat. 100: 419-424.

Hurlbert, S. H. 1978. The measurement of niche overlap and some relatives. Ecology 47: 805-816.

Hyndes, G.A., M.E. Platell \& I.C. Potter. 1997. Relationships between diet and body size, mouth morphology, habitat and movements of six sillaginid species in coastal waters: implications for resource partitioning. Mar. Biol. 128: 585-598.

Hyslop, J.E. 1980. Stomach contents analysis. A review of methods and their application. J. Fish Biol. 17: 411-429.

Keen, M.A. 1971. Sea Shell of tropical west America. Marine Molluscs from Baja California to Peru. Stanford University, Stanford, California, EEUU.

Krebs, C.J., 1999. Ecological Methodology, AddisonWelsey, Menlo Park, California, EEUU.

Langton, R.W. 1982. Diet overlap between the Atlantic cod Gadus morhua, silver hake Merluccius bilinearis and fifteen other northwest Atlantic finfish. Fish. B-NOAA 80: 745-759.

Levins, R. 1968. Evolution in changing environments: some theoretical explorations. Princeton University, Princeton, Nueva Jersey, EEUU.

Link, J.S., W.T. Stockhausen \& E.T. Methratta. 2006 Food-web theory in marine ecosystems, p. 98-113. In A. Belgrano, U.M. Scharler, J. Dunne \& R.E. Ulanowicz. Aquatic food webs. An ecosystem approach. Oxford University, Oxford, UK.

Meyer, C. G., K.N. Holland, B. M. Wetherbee \& C. G. Lowe. 2001. Diet, resource partitioning and gear vulnerability of Hawaiian jacks captured in fishing tournaments. Fish. Res. 53: 105-113.

Morales-Jauregui, M.Y. 2007. Componentes tróficos de la Chanana (Haemulon flaviguttatum) de la Bahía de Navidad, Jalisco. Tesis de Licenciatura, Instituto Tecnológico de Mar No. 6, Bahía de Banderas, Nayarit, México.

Muñoz, A.A. \& F.P. Ojeda. 1998. Guild structure of carnivorous intertidial fishes of the Chilean coast: implications of ontogenetic dietary shifts. Oecologia 114: 563-573.

Pacheco, S. P. 1991. Pacífico tropical mexicano. Oceanografía física. Oceanografía de mares mexicanos. AGT, México, D.F., México.

Pinkas, L., M. S. Oliphant \& L. K. Iverson. 1971. Food habits of albacore, bluefin tuna, and bonito in Californian waters. Calif. Dep. Fish. Game. Fish Bull. 152: $1-105$

Pratchett, M.S. \& M.L. Berumen. 2008. Interespecific variation in the distributions and diets of coral reef butterflyfishes (Teleostei: Chaetodontidae). J. Fish Biol. 73: 1730-1747.

Pratchett, M.S., S.K. Wilson, M.L. Berumen \& M.I McCormick. 2004. Sub-lethal effects of coral bleaching on an obligate coral feeding butterflyfish. Coral Reefs 23: $352-356$.

Raymundo-Huizar, A.R. 2000. Análisis de la dieta de los peces demersales de fondos blandos en la plataforma continental de Jalisco y Colima, México. Tesis de Maestría, Universidad de Colima, Colima, México.

Rojo-Vázquez, J.A., B. Aguilar-Palomino, V.H. GalvánPiña, E. Godínez-Domínguez，S. Hernández-Vázquez, S. Ruiz-Ramírez \& G. Lucano-Ramírez. 2001. Ictiofauna de la pesquería ribereña en Bahía Navidad, Jalisco, México asociada al evento El Niño19971998. Rev. Biol. Trop. 49: 841-854.

Rojo-Vázquez, J.A., C. Quiñonez-Velázquez, H.A. Echavarria-Heras, G. Lucano-Ramírez, E. GodínezDomínguez, S. Ruiz-Ramírez, V.H. Galván-Piña \& O. Sosa-Nishizaki. 2008. The fish species composition and variation of catch from the small-scale gillnet fishery before, during and after the 1997-1998 ENSO event, central Mexican Pacific. Rev. Biol. Trop. 56: 133-152.

Ross, S.T., 1986. Resource partitioning in fish assemblages: a review of field studies. Copeia: 352-388.

Salazar-Vallejo, S.I., J.A. León-González \& H. SalaicesPolaco. 1989. Poliquetos (Annelida: Polychaeta) de 
México. Libros Universitarios. Universidad Autónoma de Baja California Sur, Baja California Sur, México.

Santamaría-Miranda, A., M. Saucedo-Lozano, M.N. Herrera-Moreno \& J.P. Apún-Molina. 2005. Hábitos alimenticios del pargo amarillo Lutjanus argentiventris y del pargo rojo Lutjanus colorado (Pisces:Lutjanidae) en el norte de Sinaloa, México. Rev. Biol. Mar. Oceanogr. 40: 33-44.

Saucedo-Lozano, M. \& X. Chiappa-Carrara, 2000. Alimentación Natural de Juveniles de Lutjanus guttatus
(Pices:Lutjanidae) en la costa de Jalisco y Colima, México. Bol. Centro Invest. Biol. 34: 81-304.

Suárez-Morales, E., C. Franco-Gordo \& M. SaucedoLozano. 2000. On the pelagic copepod community of the central Mexican tropical Pacific (Autumn, 1990). Crustaceana 73: 751-761.

Vázquez, R.I., J. Rodríguez, L.A. Abitia \& F. Galván. 2008. Food habits of the yellow snapper Lutjanus argentiventris (Peters, 1869) (Percoidei: Lutjanidae) in La Paz Bay, Mexico. Rev. Biol. Mar. Oceanogr. 43: 295-302. 
